\section{mCRPC: neue Behandlungsoptionen vonnöten}

- Patienten mit einem kastrationsresistenten und metastasierten Prostatakarzinom ( $m C R P C)$ haben eine schlechte Prognose und versterben überwiegend an den Krebsfolgen. Bislang gibt es in diesem späten Tumorstadium nur wenige Behandlungsoptionen. Dementsprechend sehen Urologen Bedarf an neuen Therapien, die das Leben der Patienten verlängern können. Das ergab eine Umfrage unter 500 Urologen und Onkologen aus den fünf einwohnerreichsten EU-Ländern, die Dr. Ian Banks, London, präsentierte. Die befragten Ärzte gaben als wichtigstes Merkmal einer neuen Substanz das Gesamtüberleben an, gefolgt von einer besseren Verträglichkeit für den Patienten. Für Patienten ist dagegen am wichtigsten, ihren Lebensstil während der Therapie weitgehend aufrecht erhalten zu können und in den Entscheidungsprozess über die richtige Therapie einbezogen zu werden. Ärzte sollten das bei der Wahl der Therapie berücksichtigen, empfahl Banks. „Oft informieren wir Patienten über Dinge, die sie unserer Meinung nach wissen sollen - statt über Dinge, die sie wissen wollen."

Welche Optionen für Patienten mit fortgeschrittenem mCRPC in Zukunft bestehen könnten, fasste Prof. Axel Heidenreich, Aachen, zusammen. Unter anderem stellte er MDV3100 vor, einen neuen oralen Androgenrezeptor-Signal-Inhibitor, der den Androgenstoffwechsel an drei Angriffspunkten inhibiert: Er verhindert, dass Testosteron an den Androgenrezeptor bindet, blockiert die Translokation in den Nukleus und dort die Bindung an die DNA. Einerseits wird so das Tumorwachstum gebremst, andererseits die Apoptose der Krebszellen induziert. In der randomisierten doppelblinden multizentrischen Placebo-kontrollierten Phase-III-Studie AFFIRM erhielten 1.199 Patienten, du zuvor mit einer Docetaxel-haltigen Chemotherapie behandelt wurden, entweder $160 \mathrm{mg}$ MDV3100 oder Placebo. Unter MDV3100 hatten Patienten einen signifikanten Überlebens- vorteil von fast fünf Monaten (18,4 vs. 13,6 Monaten, $p<0,0001$ ). Auch alle sekundären Endpunkte waren signifikant, unter anderem konnte bei 54,0\% der Patienten (Placebo: $1,5 \%)$ der PSA-Wert um mehr als die Hälfte reduziert werden. Die Nebenwirkungen des Verums waren mit denen von Placebo vergleichbar. „In Zukunft müssen wir herausfinden, in welcher Reihenfolge welcher Patient mit welcher Substanz nach Versagen der Androgendeprivationstherapie am besten behandelt wird", betonte Heidenreich. MDV3100 könne dabei eine wichtige Rolle spielen. Derzeit laufen noch weitere Studien: Unter anderem werden Wirkung und Sicherheit von MDV3100 bei chemonaiven mCRPC-Patienten (PREVAIL-Studie) sowie in einer Phase-II-Studie bei Patienten mit metastasiertem Prostatakarzinom gegenüber Bicalutamid untersucht. Mit einer Zulassung ist laut Heidenreich etwa Anfang 2013 zu rechnen. Sebastian Lux

Science Writers Workshop „Rising to the unmet need in advanced prostate cancer across Europe" im Rahmen des 27. EAU-Kongresses.

Paris, 26. Februar 2012

Veranstalter: Astellas, München

\title{
Händedesinfektion: Standardeinreibemethode nicht mehr überzeugend
}

_ Im Vergleich zur jahrelang empfohlenen und praktizierten mehrstufigen Einreibemethoden führen individuelle, freie Techniken zu deutlich besseren Ergebnissen bei der Desinfektion der Hände. Zu diesem Ergebnis kommt eine Studie [Kampf G et al., BMC Infect Dis 2008; 8: 149], die Studienleiter Prof. Dr. Günter Kampf, Hamburg, präsentierte. Verglichen wurde das seit 30 Jahren als Standard empfohlene Einreibeverfahren, bei dem sechs Schritte der Händedesinfektion fünf Mal wiederholt werden, mit eigenverantwortlichen Methoden, die ohne spezielle Vorgaben für die Anwender auskommen. Verwendet wurde ein Desinfektionsmittel, das einen fluoreszierenden Farbstoff enthielt, der unter UV-Licht die Benetzung der Hände sichtbar macht. Das überraschende Ergebnis: Die Standardmethode schnitt unter allen Techniken am schlechtesten ab. Bei einmaliger Anwendung blieben $100 \%$ der Hände unvollständig behandelt und selbst bei fünfmaliger
Wiederholung und einer Anwendungsdauer von durchschnittlich 37 Sekunden noch zwei Drittel der Hände, die damit eine potenzielle Gefahr für nosokomiale Infektionen darstellten. Unter der freien Methode mit einer Dauer von 28 Sekunden blieb nur knapp die Hälfte nicht vollständig benetzt. Für Bereiche wie Fingerkuppen und Daumen, die für die Greiffunktion wichtig sind, sowie die Handinnenfläche war die Desinfektion ohne Vorgaben sicherer.

"Die freie Technik vereinfacht und verbessert die Handhygiene qualitativ, steigert die Compliance der Anwender und damit die Sicherheit der Patienten", erläuterte Kampf. Vor diesem Hintergrund empfiehlt die „Aktion Saubere Hände" seit Ende 2011 die eigenverantwortliche Methode als neuen Standard. Träger der „Aktion Saubere Hände", die vom Bundesgesundheitsministerium unterstützt wird, sind das Referenzzentrum für die Surveillance nosokomialer Infektionen, das Aktionsbündnis Patientensi- cherheit und die Gesellschaft für Qualitätsmanagement im Gesundheitswesen.

Michael Koczorek

Symposium „Krankenhausinfektionen und Händehygiene - wie neue Forschungsergebnisse den Infektionsschutz verbessern",

Berlin, 9. Februar 2012,

Veranstalter: Bode Science Center, Hamburg

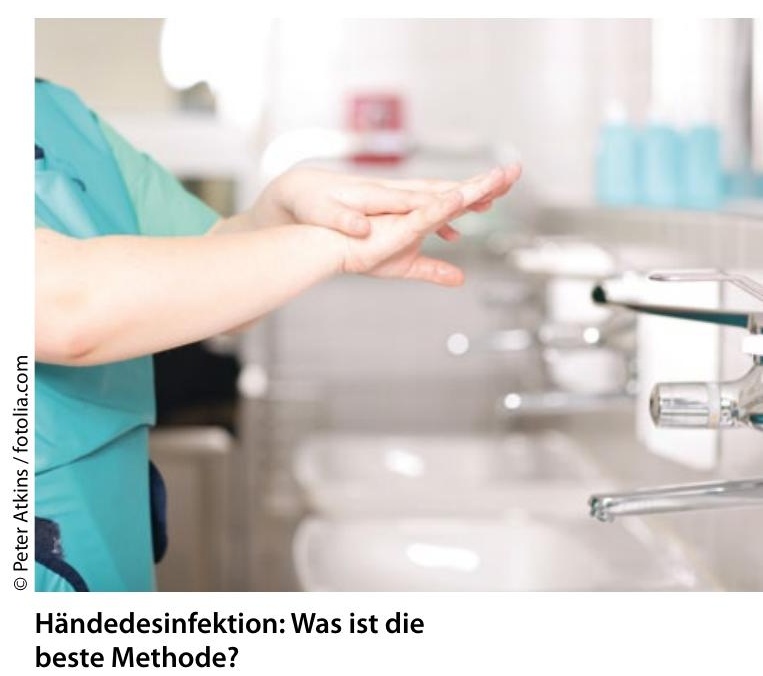

